\title{
Communication Technology and Application of Seismic Precursor Network Instrument
}

\author{
Jianguo Wang, Wei Wang, Huiqin Yao, Xun Gao, Mingdong Zhang \\ Earthquake Administration of Tianjin Municipality, Tianjin, China, 300201 \\ Email: tjjgw@163.com
}

Received 2012

\begin{abstract}
In this paper, the communication technology of seismic precursor network instrument is introduced, including instruction format and returned information format of instrument login, status information acquisition, and current measured data acquisition. The remote monitoring alarm software is based on this technology, and also introduced that the structure of monitoring information table, abnormal alarm index, and monitoring strategy. The application of the software raises instrument running rate and observation data quality.
\end{abstract}

Keywords: Network Instrument; Communication Technology; Remote Monitoring Alarm

\section{Introduction}

Through digital seismic observation network projects, Tianjin seismic precursor network, from network to the station, distributed database to station, IP to device can monitor and control precursor observation instrument remotely. Realized gathering instrument data and realtime monitoring instrument's status and property, by which remote change the parameters and adjust the working status of instrument, and can control basic instrument calibration [1-3].

During seismic precursor network instrument running, always following system halted, clock error and wrong data and so on, having a bad effect on instrument running rate and data quality.

For this reason, according stipulation on geoscience observatory network in China, we compiled the software to monitor network instrument remotely and alarm by C\#, to identify and solve problems in time and improve running rate and data quality of instrument [1-3].

\section{Network Instrument Communication Technology}

Stipulation on geoscience observatory network in China is a network communication interface enacted for precursor observation instrument. It detailed prescribes the precursor network instrument's communication interface, device information, running function, data format, communication protocol and access security control protocol, etc. It is an essential communication protocol for network precursor instrument [1-3].

The data communication of client and instrument is conducted by Ethernet and Socket based on TCP/IP protocol. Client sends request to instrument, realizes the control of instrument and exchange of data information. Instrument accepts and responds the instruction from client, then implements the corresponding action to complete the data information exchange with client; instrument can be regard as server [1-3].

System. Net. Sockets namespace in C\# provides related class for writing network application programs like Socket, TcpClient, and Net Work Stream and so on.

Socket class is used in management connection, to realize Berkeley sockets interface. TcpClient class is based on the Socket class, allows creating and using TCP connection. Net Work Stream class deals specifically with data stream of network form, receipts object parameter of Socket class, and operate network data access [1-3].

\subsection{Detect Instrument Network Status}

Using C\# call ping command (ping -n 1 192.168.1.2) to detect connection of network, it is an easy way.

In the output stream, "( $0 \%$ loss $)$ " indicates that the network is smooth, "Ping request could not find host" indicates that ping request could not find host, "Request timed out or (100\% loss)" indicates ping request timed out.

\subsection{Login Instrument}

Client and instrument establish connection through instruction mode, according to user name and password authenticates identity [1-3].

Send instruction format: get /length+ID+lin+username 
+password /http/1.1

Among them, length: the instruction length word; ID: instrument serial number; lin: user login command word; username: user name; password: password.

Return message format: If the login and connect is normal, return \$ackln; if the login and connect is abnormal, return \$nakln; if the instrument receive the erroneous instruction, return \$errın.

Instrument login code:

// Namespace reference

using System. Net. Sockets;

// Instrument IP address

string IP= "192.168.1.2";

// Instrument port

string Port="81";

// Instrument login instruction

string Instruction="get/42+X311JSEA0003+lin+username +password /http/1.1";

// Create a TcpClient, connected to the instrument.

TcpClient Client $=$ new TcpClient (IP, Port);

// Get client stream for reading and writing.

NetworkStream Network_Stream $=$ Client.GetStream ();

// Translate the instruction message into ASCII and store it as byte array.

byte[] byteCode = Encoding. ASCII. Get Bytes (Instruction);

// Send the instruction message to the connected TcpServer.

Network_Stream. Write (byte Code, 0, byte Code. Length);

// Store the response bytes in the buffer.

byte[] buffer = new byte[1024];

// Read the first batch of the TcpServer response bytes.

int result $=$ Network_Stream. Read (buffer, 0, buffer. Length);

if (Encoding. ASCII. GetString(buffer, 0, result). Trim(). ToLower() == "\$ack")

\{

// Instrument login successful

// Get instrument status information

// Get current measured data

\}

\subsection{Get instrument Status Information}

Instrument status information includes instrument running environment, running results, abnormalities and various controllable status information [1-3].

Send instruction format: get /length+ID+ste /http/1.1

Among them, ste: the command word to get instrument status information.

Return message format: \$message length of status information data packetln content of status information data packet $\backslash n$ ack $\backslash n$
Content of status information data packet: length, instrument clock, clock status, instrument zero, DC power supply status, AC power supply status, self-calibration switch status, zero switching status, the number of events triggered, abnormal alarm status and custom status.

The content and format of instrument status information sees Table 1.

For example:

Send instruction to get instrument status information: get /19+X311JSEA0003+ste /http/1.1

Return data packet as following:

$\$ 39$

392010081614500910.0000000000

ack

\subsection{Get Current Measured Data}

The current measured data is the latest 5-minutes sample. If there is no sampling, get a recent sample [1-3].

Send instruction format: get /length+ID+dat+5/http/ 1.1

Among them, dat: data transfer command word.

Return message format: \$message length of data packet ln content of data packet $\backslash n$ ack $\backslash n$

Table 1. The content and format of instrument status information.

\begin{tabular}{|c|c|}
\hline Status information & explanation \\
\hline Length & $\begin{array}{l}\text { Record all the bytes contained in the } \\
\text { status information }\end{array}$ \\
\hline Instrument clock & $\begin{array}{l}\text { Using the "YYYYMMDDHHMMSS" } \\
\text { (yearmonthdayhourminutesecond) } \\
\text { format }\end{array}$ \\
\hline Clock status & $\begin{array}{l}\text { "0" means GPS time service, " } 1 \text { " means } \\
\text { SNTP time service, "2" means internal } \\
\text { clock }\end{array}$ \\
\hline Instrument null point & Custom real number \\
\hline DC power status & "0" means normal, "1" means abnormal \\
\hline AC power status & "0" means normal, "1" means abnormal \\
\hline Self-calibration switch status & "0" means close, "1" means open \\
\hline Zero switching status & "0" means close, "1" means open \\
\hline Number of events triggered & $\begin{array}{l}\text { The number of day events have been } \\
\text { triggered }\end{array}$ \\
\hline Abnormal alarm status & $\begin{array}{l}\text { One char, the corresponding } 8 \text { bits } \\
\text { from high to low represent power } \\
\text { failure, clock exception, unauthorized } \\
\text { access, event trigger, storage exception, } \\
\text { abnormal measured data, and custom } \\
\text { alert status }\end{array}$ \\
\hline Custom Status & $\begin{array}{l}\text { Two chars } 16 \text { bit, each bit’s "0" or "1" } \\
\text { represent custom content }\end{array}$ \\
\hline
\end{tabular}


Content of data packet: length, start time, station code, instrument ID, sample rate, item number, item code 1 , item code $2, \ldots$, item code $n$, current 5 minutes observation data sorted by item order and sampling order.

In which, the length is byte count of the entire data block, missing data is null.

For example:

Send instruction to get instrument current measured data: get /21+X311JSEA0003+dat+5/http/1.1

Return data packet as following:

$\$ 189$

18914480012001 X311JSEA0003 010331273124

$\begin{array}{llllll}3125 & 54004.5 & 28502.9 & -0009.67 & 54004.6 & 28503.6\end{array}$

$\begin{array}{llllll}-0009.77 & 54005.0 & 28504.2 & -0009.78 & 54004.9 & 28504.1\end{array}$

-0009.76 54004.5 28504.6-0009.84

ack

\section{Network Instrument Monitoring Alarm Software}

Client monitoring alarm software finds out instrument abnormal, sounds alarm and writes the instrument status information and current measured data into oracle $10 \mathrm{~g}$ distributed database, by detecting instrument network status, collecting instrument status information and current measured data [4-6].

The work flow chart of remote monitoring alarm software sees Figure 1.

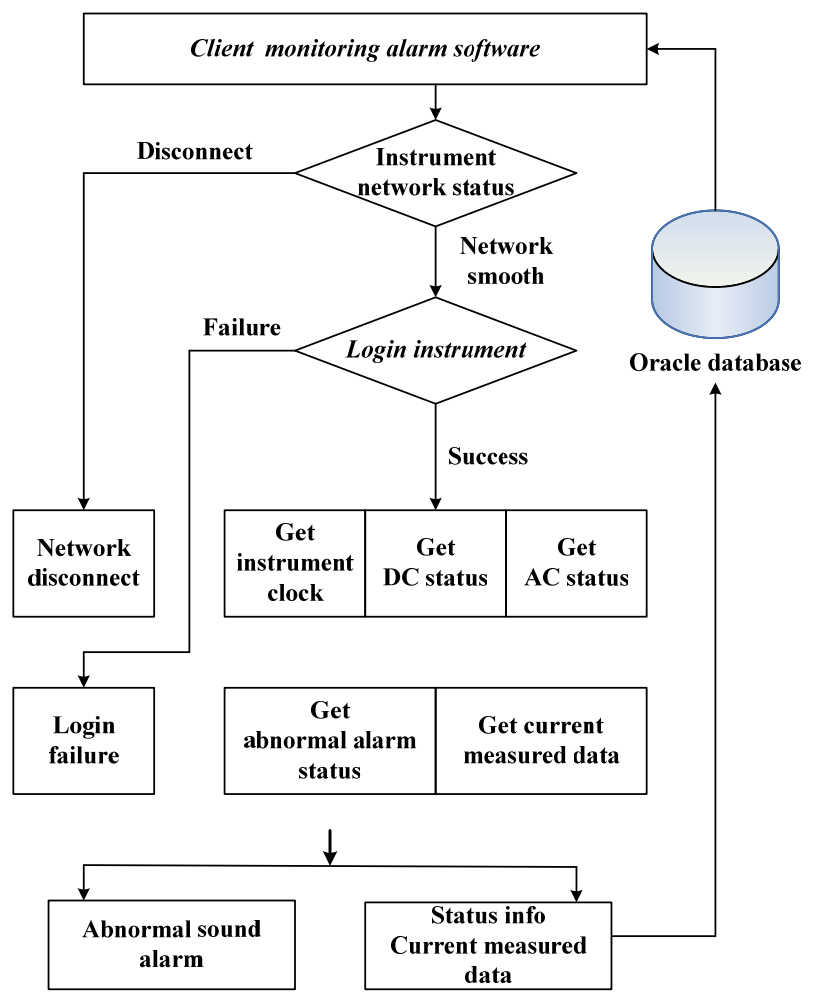

Figure 1. The work flow chart of remote monitoring alarm software.

\subsection{Design the Structure of Instrument Monitoring Information Table}

In the oracle database, the instrument monitoring information table "Monitor" is used to store instrument status information and current measured data.

The table structure sees Table 2 [7].

\subsection{Abnormal Alarm Index}

When the network instrument has the abnormal situation as following, client software will sound alarm.

Ping instrument host, if return "Ping request could not find host, Request timed out or (100\% loss)”, indicate that the instrument is no network.

Instrument clock and client computer clock have a difference of more than 3 minutes, then need to adjust instrument clock.

For example: On August 30, 2010, the operator on duty found that $\mathrm{Xu}$ Zhuangzi wyy-1 meteorological instrument was faster 4 minutes than client computer, then entered the instrument web page 192.168.1.3, manually modified the instrument clock and restarted it. Note: client computer calibrates inner clock through time server 192.168.1.4.

Instrument DC power status is 1 , indicates abnormal presence, such as: DC power failure.

Instrument AC power status is 1 , indicates abnormal presence, such as: AC power failure.

Table 2. The structure of instrument monitoring information table.

\begin{tabular}{lccc}
\hline \multicolumn{1}{c}{$\begin{array}{c}\text { Field English } \\
\text { Name }\end{array}$} & $\begin{array}{c}\text { Field Chinese } \\
\text { Name }\end{array}$ & $\begin{array}{c}\text { Prime } \\
\text { Key }\end{array}$ & $\begin{array}{c}\text { Field } \\
\text { Type }\end{array}$ \\
\hline STARTDATE & Client Clock & Yes & DATE \\
UNITCODE & Unit Code & Yes & VARCHAR2(5) \\
STATIONID & Station ID & Yes & CHAR(5) \\
POINTID & Point ID & Yes & CHAR(1) \\
ITEMID & Item ID & Yes & CHAR(4) \\
INSTRUMENTIP & Instrument IP & & VARCHAR2(50) \\
UNITNAME & Unit Name & & VARCHAR2(50) \\
STATIONNAME & Station Name & & VARCHAR2(50) \\
INSTRUMENTNAME & Instrument Name & & VARCHAR2(50) \\
NETWORKSTATUS & Network Status & & VARCHAR2(50) \\
INSTRUMENTTIME & Instrument Clock & & DATE \\
INTERVALMINUTE & Interval Minute & & NUMBER(20) \\
DCSTATUS & DC Status & & VARCHAR2(50) \\
ACSTATUS & AC Status & & VARCHAR2(50) \\
ALARMSTATUS & Alarm Status & & VARCHAR2(50) \\
ITEM & Current Item & & VARCHAR2(50) \\
DATA & Current Data & & NUMBER(38,4) \\
\hline & & &
\end{tabular}


Instrument abnormal alarm status takes a byte, the corresponding 8 bits from high to low represent power failure, clock exception, unauthorized access, event trigger, storage exception, abnormal measured data, and custom alert status. The any position is 1 , indicates corresponding abnormal presence.

Using aggregate functions "max, min, sum, count", and group statement "group by", to count measured data that has been put in storage, to get max value, min value, variation amplitude and average value [8,9].

If the variation amplitude is over than threshold, that means abnormal situation. Such as well water level exceeds $1 \mathrm{~m}$, well water temperature or auxiliary temperature of geomagnetic observation room exceeds $1^{\circ} \mathrm{C}$, total intensity of geomagnetic relative record, vertical component or horizontal component exceeds $20 \mathrm{nT}$, air temperature exceeds $20^{\circ} \mathrm{C}$, atmospheric pressure exceeds 30 hPa and so on[8,9].

SQL statement as following:

Select unitcode, stationid, pointid, itemid,..., instrumentitem, max (instrumentdata), min(instrumentdata), sum(instrumentdata), count (instrumentdata) from qzprocess. monitor where startdate between to_date ('2010-8-1','yyyy-mm-dd') and to_date ('2010-8-31', 'yyyy-mm-dd') group by unitcode, stationid, pointid, itemid,..., instrumentitem order by unitcode, stationid, pointid, itemid.

\subsection{Monitoring Strategy}

Monitoring methods include manual mode and automatic mode. Manual monitoring may monitor network instrument at any time by click a button; automatic monitoring need to set a monitoring interval, namely, every few minutes to monitor once.

The software uses multi-threading technology, assigns a thread for each instrument, thus the execution time is foreign to the number of instrument, reducing software running time and improving software efficiency.

\section{Conclusions}

Using seismic precursor network instrument communication technology and oracle distributed database reading and writing technology, we have developed monitoring alarm software, which catches the instrument abnormal information initiatively, makes a sound to alarm, and provides the technology support for finding and solving instrument's problem, and improves the instrument's running rate and data quality effectively, and enhances the Tianjin seismic precursor network running management level $[8,9]$.

On appraisal of national earthquake precursor network, Earthquake Administration of Tianjin Municipality obtained the first place on "System Running" and the second place on “Output and Application” in 2009, obtained the first place on "System Running" and the first place on "Output and Application” in 2010, obtained the second place on "System Running" and the first place on “Output and Application” in 2011, achieved excellent results, and produced good social benefits.

\section{Acknowledgements}

This research was supported in part by methods research and application for operation and management of regional seismic precursor network in Tianjin under Grant No. XH12004 and in part by earthquake precursor monitoring network and data management platform for research based on WebGIS under Grant No. 09ZCKFSF00700 and in part by Tianjin seismic safety foundation engineering "precursor instrument renovation project”.

\section{REFERENCES}

[1] China Earthquake Administration, "Stipulation on Geoscience Observatory Network in China,” Beijing: Seismological Press, 2005.

[2] X. Y. Wang, Z. A. Zhou and H. Ding, "Application of Network Communication Technology in Seismic Precursor Instruments,” North China Earthquake Sciences, Vol. 23, No. 4, 2005,pp. 26-30.

[3] X. Y. Wang, Z. A. Zhou and A. C. Liu, "Discussion on Application of Stipulation of Network Communication for Earthquake Precursory Observation Devices,” Journal of Geodesy and Geodynamics, Vol. 28, No. 4, 2008, pp. 131-135.

[4] X. Y. Wang, Z. A. Zhou and A. C. Liu, "Design and Implementation of Dynamic Monitoring and Alerting Functions for the Earthquake Precursory Observation Devices,” Journal of Seismological Research, Vol. 32, No. 4 , 2009, pp. 431-435.

[5] K. C. Zhou, Z. X. Li and S. Wang, "Monitoring and Managements System for Earthquake Precursory Data," Earthquake, Vol. 26, No. 1, 2006, pp. 115-122.

[6] J. G. Wang, X. F. Cui and H. -R. Chen, "A Software System for Precursor Observation Data Monitoring and Anomaly Auto-identifying," Journal of Seismological Research, Vol. 30, No. 1, 2007, pp. 83-87.

[7] K. -C. Zhou, C. -H. Jiang and S. -W. Ji, "On the Design of Earthquake Precursor Observation Database System,” Earthquake, Vol. 30, No. 2, 2010,pp. 143-151.

[8] J. -G. Wang, H. -J. Dong and X. -G. Xu, “Development of the Software for Operational Management of Tianjin seismic Precursor Station Network Built during the Tenth Five-Year Plan," Seismological and Geomagnetic Observation and Research, Vol. 30, No. 3, 2009, pp. 114-120.

[9] J. -G. Wang, H. -Q. Yao and X. Gao, "Management of running, Monitoring and Maintenance of TianJin Earthquake Precursor Network," Journal of Geodesy and Geodynamics, Vol. 30, 2010, pp. 111-115. 